\title{
Assessment of Alveolar Bone Mineral Density as a Predictor of Lumbar Fracture Probability
}

\author{
Yoshitomo Takaishi - Seizaburo Arita - Mitsugi Honda • \\ Takeshi Sugishita $\cdot$ Aiko Kamada $\cdot$ Takashi Ikeo • \\ Takami Miki $\cdot$ Takuo Fujita
}

To view enhanced content go to www.advancesintherapy.com Received: January 31, 2013 / Published online: May 15, 2013

(C) Springer Healthcare 2013

\section{ABSTRACT}

Introduction: Osteoporosis and tooth loss have been linked with advancing age, but no clear relationship between these conditions has been proven. Several studies of bone mineral density

Y. Takaishi $(\bowtie) \cdot$ T. Sugishita

Takaishi Dental Clinic, Hojo-guchi, Himeji, Japan e-mail: takaishi@dentalgraphic.com

Y. Takaishi · A. Kamada · T. Ikeo

Department of Biochemistry, Osaka Dental

University, Hanazonocho, Hirakata, Japan

Y. Takaishi · T. Miki

Department of Geriatric Medicine,

Osaka City University, Abeno-ku, Osaka, Japan

S. Arita

Department of Life and Medical Sciences,

Doshisha University, Tatara, Kyotanabe, Japan

M. Honda

Department of Radiology, Okayama University

Hospital, Kita-ku, Okayama, Japan

T. Fujita

Katsuragi Hospital, Kishiwada, Japan

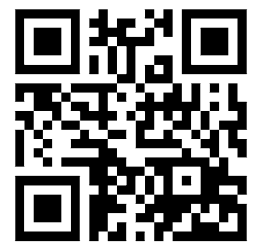

Enhanced content for Advances in Therapy articles is available on the journal web site: www.advancesintherapy.com measurements of the jaw and spine have shown similarities in their rate of age-related deterioration. Thus, measurements of jawbone density may predict lumbar vertebral bone density. Using jawbone density as a proxy marker would circumvent the need for lumbar bone measurements and facilitate prediction of osteoporotic spinal fracture susceptibility at dental clinics. We aimed to characterize the correlation between bone density in the jaw and spine and the incidence of osteoporotic spinal fractures.

Methods: We used computerized radiogrammetry to measure alveolar bone mineral density (al-BMD) and dual-energy $\mathrm{X}$-ray absorptiometry to measure lumbar bone mineral density (L-BMD). L-BMD and al-BMD in 30 female patients (average age: $59 \pm 5$ years) were correlated with various patient attributes. Statistical analysis included area under the curve (AUC) and probability of asymptomatic significance (PAS) in a receiver operating characteristic curve. The predictive strength of L-BMD T-scores (L-BMD[T]) and al-BMD measurements for fracture occurrence was then compared using multivariate analysis with category weight scoring. 
Results: L-BMD and al-BMD were significantly correlated with age, years since menopause, and alveolar bone thickness. Both were also negatively correlated with fracture incidence. Category weight scores were -0.275 for a L-BMD(T) $<80 \%$; +0.183 for a L-BMD(T) $\geq 80 \%$; -0.860 for al-BMD $<84.9$ (brightness); and +0.860 for al-BMD $\geq 84.9$. AUC and PAS analyses suggested that al-BMD had a higher association with fracture occurrence than L-BMD.

Conclusions: Our results suggest the possible association between al-BMD and vertebral fracture risk. Assessment of alveolar bone density may be useful in patients receiving routine dental exams to monitor the clinical picture and the potential course of osteoporosis in patients who may be at a higher risk of developing osteoporosis.

Keywords: Alveolar; Bone mineral density; Computerized; Fracture; Lumbar; Osteoporosis; Periodontitis; Predictive; Radiogrammetry

\section{INTRODUCTION}

Osteoporosis is a systemic disease characterized by reduced bone mass and a thinning of the trabecular architecture that often leads to increased bone fragility and subsequent fracture $[1,2]$. A primary cause of osteoporosis in women arises from a drop in estrogen levels after menopause. This drop in estrogen levels has also been associated with an increase in the loss of teeth and resorption of alveolar bone $[3,4]$. Osteoporosis is detected with the use of $\mathrm{X}$-ray and other more advanced methods, such as dual-energy X-ray absorptiometry (DXA). However, osteoporosis is rarely detected in patients prior to their first fracture. With an aging population, this will likely place a substantial burden on the medical system in future years. Therefore, it will be important to be able to detect osteoporosis before the occurrence of fracture.

The first link between osteoporosis and bone loss in the oral cavity was established by Groen et al in the 1960s [5]. The jawbone, despite its uniquely discrete location and function, remains part of the skeletal system and is metabolically regulated along with other aspects of the skeleton, including the vertebrae and long bones-sites frequently prone to osteoporotic fracture. The association between osteoporosis and tooth loss is still a contentious issue because of mixed results in the literature, and studies have yet to show conclusively an association between osteoporosis and the loss of periodontal attachment, the loss of teeth, and/or changes to the residual ridge. Two studies by Krall et al. [3, 4] and another by Taguchi et al. [6] found positive associations between tooth loss and reduced bone mineral density (BMD) in postmenopausal women. Moreover, recent work has established a link between tooth loss, periodontal disease, and osteoporosis [7-11]. However, others failed to report similar associations for both periodontal disease and tooth loss with BMD measurements [12, 13]. Consequently, elucidating the relationship between oral health and osteoporosis is still an important clinical research focus in the field of dentistry.

Various techniques have been used to assess BMD within the mandible, including DXA, quantitative computed tomography (qCT) and radiographic absorptiometry, as well as standard intraoral radiographs and panoramic radiographs employed in dental clinics [9]. These techniques are used to detect changes in the normal anatomy and bone density of the jaw by measuring aspects such as alveolar crest height [8], mandibular trabecular pattern [14, 15], buccolingual distance of the alveolar process [16], and mandibular cortical indices [17-21]. 
An increasing number of studies have examined BMD changes in the mandible. A study by Pluskiewicz et al. [22] in 2000 assessed the relationship between BMD in the mandible, hip, calcaneus, and hand phalanges in 36 female subjects. They found that BMD of the mandible significantly decreased with years since menopause (YSM), age, and body size in a stepwise multiple linear regression analysis, concluding that the mandible may be an appropriate site for assessing $\mathrm{BMD}$ in the diagnosis of osteoporosis [22]. In addition, using ovariectomized monkeys, a study by Binte Anwar et al. [23] showed an increase in fragility of the trabecular bone in the molar alveolar bone concomitant with decreased BMD in the lumbar bone, as measured using DXA. More recently, Miliuniene et al. [24] sought to compare mandibular cortical bone height with measurements of BMD of the lumbar vertebrae in an effort to use jaw BMD for fracture prediction.

Despite these efforts, the ability to predict the incidence of osteoporotic fractures based on jawbone measurements has not been accomplished [25-27]. This is possibly due to technical limitations with measuring jaw BMD. The use of advanced methods, such as DXA and peripheral qCT, has posed serious difficulties on account of the complex and limited space around the jaw, as well as the potential risk for high local radiation exposure to neighboring vital structures, particularly in the head and neck.

A precise and accurate computed radiogrammetry method has been developed to measure dental alveolar bone (Bone Right ${ }^{\circledR}$, Dental Graphic Ltd, Himeji, Japan) that overcomes the difficulties associated with other methods [28-30]. This technique has been used extensively since 2003, procuring accurate measurements of al-BMD by pasting dental X-ray films with an aluminum wedge. Unlike the method devised by Kribbs et al. [10] for the simple analogous comparison between the wedge and the bone, the aluminum wedge film was employed for normalization and standardization of the data in this new method. The significant correlation between age and decreased measurements of al-BMD in normal ambulant subjects deemed this a promising method for the evaluation of osteoporosis and risk of fracture [28-30].

From the current literature, it is conceivable to assume that BMD measurements of the jaw could act as a predictive indicator of skeletal bone density and the potential risk of developing osteoporosis. The use of jawbone density as a proxy marker could circumvent the need for lumbar bone measurements and we hypothesize that jaw BMD measurements, taken routinely during dental examinations, may indeed be able to predict the risk of osteoporosis better than lumbar BMD measurements. In the present study, we tested the utility of the Bone Right ${ }^{\circledR}$ method for evaluating jaw BMD at a crucial site within the alveolar bone, the first right mandibular premolar tooth, which is influenced by systemic bone metabolism and the local periodontal state. In the same patients, we then compared these measurements with DXA scans of the L1-L4 lumbar vertebrae to determine the lumbar BMD T-scores (L-BMD[T]; L-BMD divided by young adult mean [YAM]), expressed as a percentage of YAM) to explore the possibility of predicting the incidence of osteoporosis-related fractures with al-BMD in patients receiving routine dental examinations.

\section{METHODS}

\section{Subjects}

Female volunteers were recruited unselectively in chronological sequence from a series of patients who had consulted a dental clinic for 
general basic dental care and prevention of periodontal disease, including dental calculi, brushing guidance, and washing and curettage of the periodontal pockets. These volunteers met the following criteria: (1) postmenopausal state; (2) an absence of endocrine or metabolic diseases (such as thyroid disease or osteoporosis) that could give rise to mineral or skeletal abnormalities; and (3) an absence of treatment for osteoporosis, such as estrogen, selective estrogen receptor modulators, or bisphosphonates or treatment with corticosteroids; (4) an absence of acute periodontal disease (negative BANA test) as a result of scaling and plaque control; this was required to artifactually overpredict systemic osteoporosis due to local periodontal bone loss.

A total of 30 postmenopausal women, between the ages of 50 and 69 years, were finally included. None of these volunteer subjects had an inflammatory disease, and the subjects did not smoke and were not habitual alcohol drinkers. A few of the subjects had a family history of fracture (Table 1). Subjects underwent various tests to determine the presence and grade of fracture (vertebral or other), the lumbar and al-BMD measurements, as well as chemical tests for markers of bone turnover. The Institutional Review Board of Katsuragi Hospital approved this study. All procedures followed were in accordance with the ethical standards of the responsible committee on human experimentation (institutional and national) and with the Helsinki Declaration of 1975, as revised in 2000. Informed consent was obtained from all patients for being included in the study.

\section{Assessment of Fractures}

Spinal fracture was found in 14 subjects and peripheral fracture in 2 . The other patients did not show evidence of fracture. History and radiography were used to assess fractures, according to the criteria suggested by Genant et al. [31]. Vertebral fractures were first graded according to this qualitative scaling to show eight patients with grade 1 (mild deformity), six patients with grade 2 (moderate deformity), and one patient with grade 3 (severe deformity). Fractures were also confirmed by a decrease in height of $3 \mathrm{~cm}$ or more and patients were classified into present (1) or absent (0) groups. A height loss of $3 \mathrm{~cm}$ was chosen as a cutoff value, because it is widely used in the clinical setting as supportive evidence of the incidence of spinal fracture. None of the subjects with spinal fracture had a history of a severe fall or evidence of infection or neoplastic invasion of the spine, suggesting that the cause of fracture in these patients was osteoporosis.

Lumbar BMD (L-BMD) was measured by dual-energy X-ray absorptiometry (DXA) (QDR 4500W, Hologic Inc., Bedford, MA, USA), expressed as a mean of the bone density measured for lumbar vertebrae L1-L4 $\left(\mathrm{g} / \mathrm{m}^{2}\right)$.

\section{Alveolar Bone Measurements}

Computed radiogrammetry of the dental alveolar bone was performed according to the Bone Right ${ }^{\circledR}$ method reported by Takaishi et al. [28-30]. Using a thin adhesive aluminum step wedge pasted on the X-ray film, pictures were taken of the regions around the first right mandibular premolar tooth, taking special care to place the X-ray tube vertical to the film. After exposure, the dental X-ray film was imported digitally using a scanner (Fig. 1). Data and histograms of the alveolar BMD (al-BMD) were recorded using dedicated software (Bone Right $\left.^{\circledR}\right)$. Figure 2 shows the measurement and calculation of al-BMD. Briefly, a line was drawn from the apex of the root parallel to the 


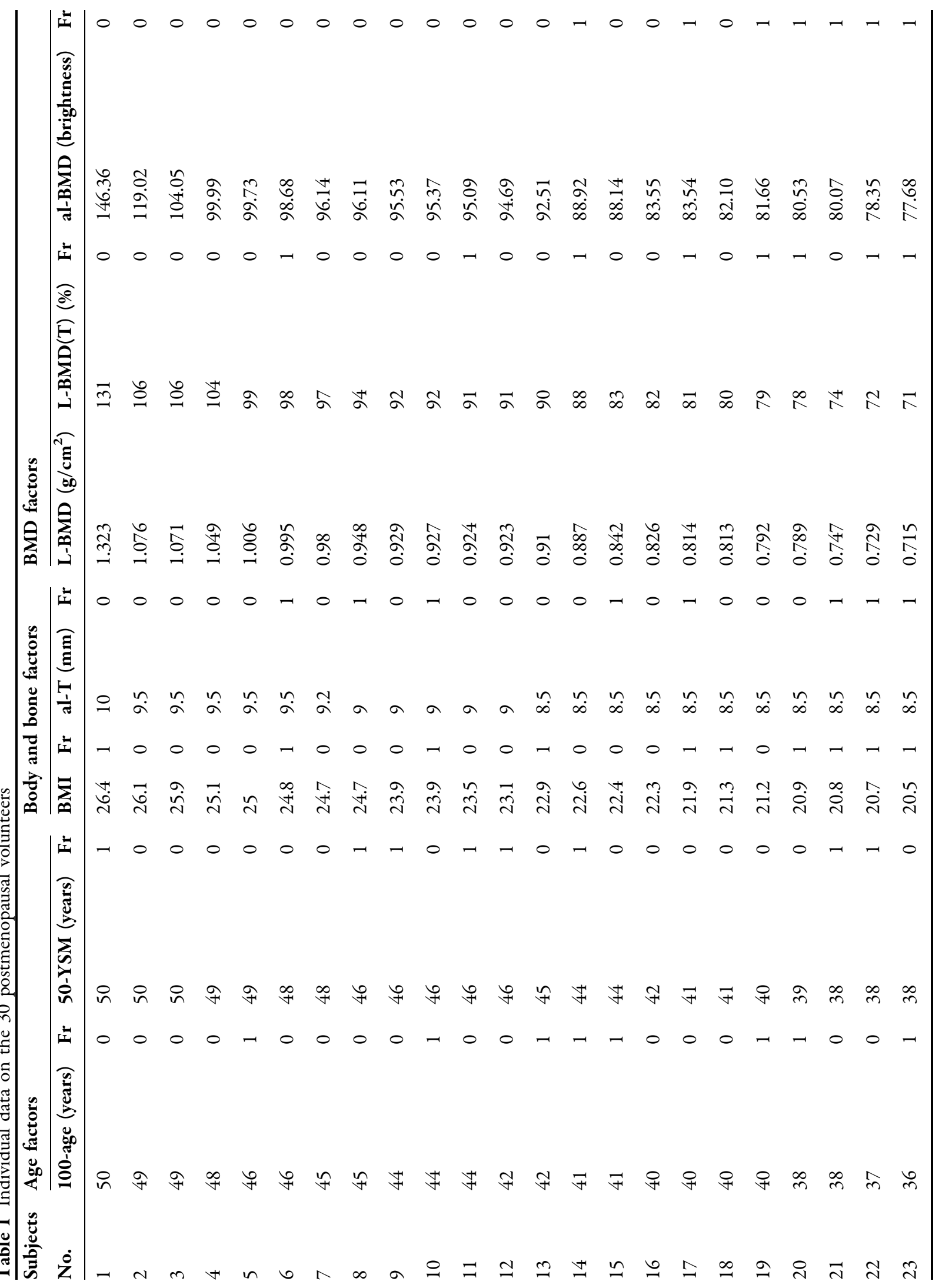




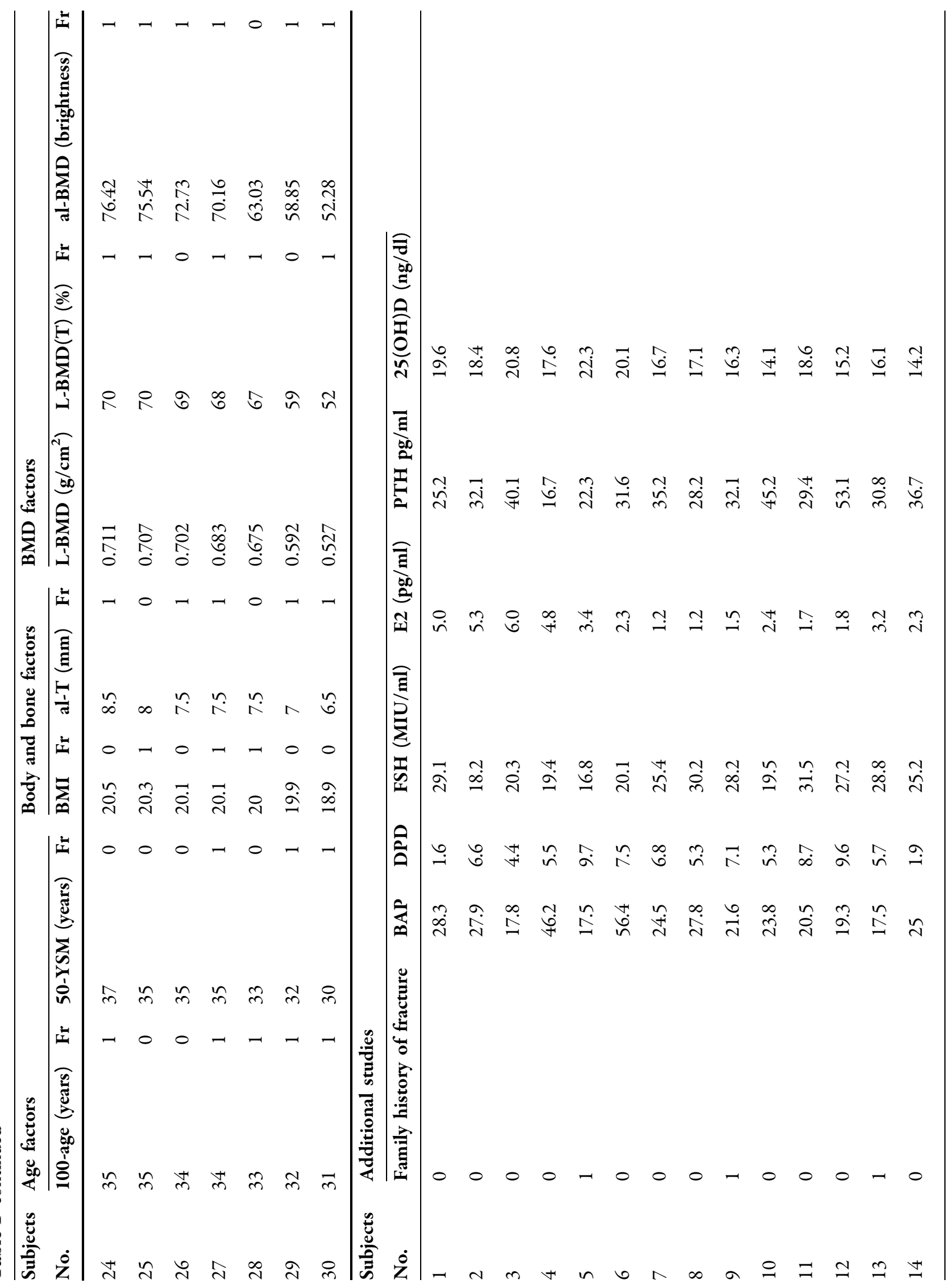




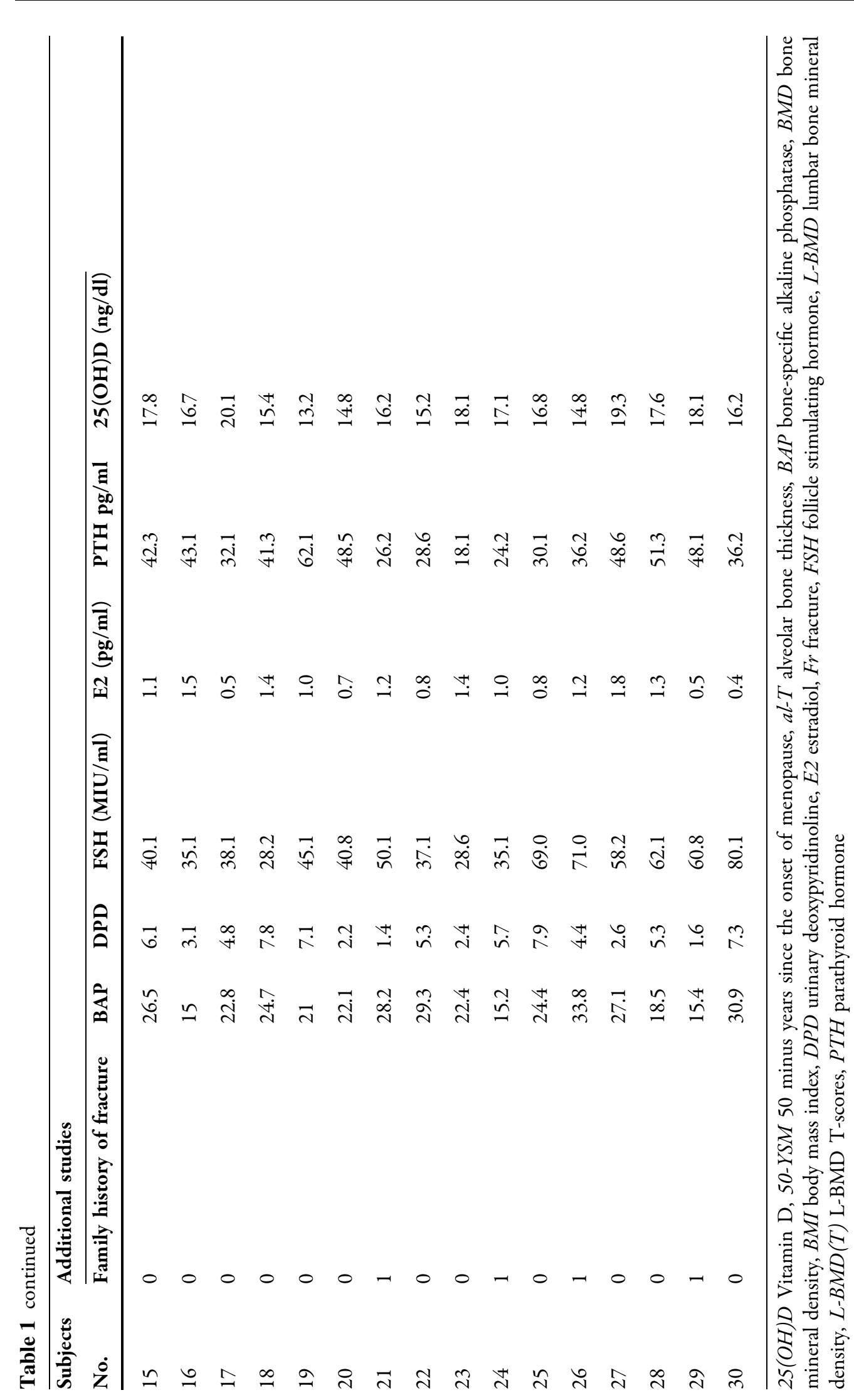


(a)

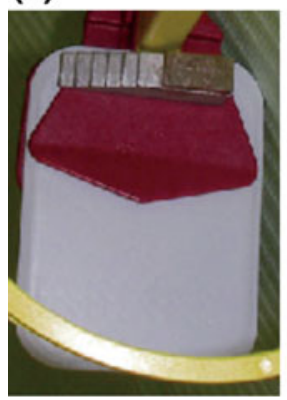

(d)

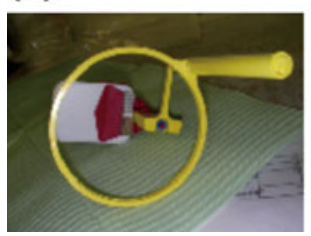

(b)

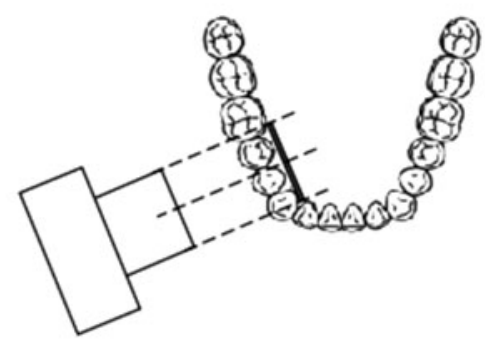

(c)
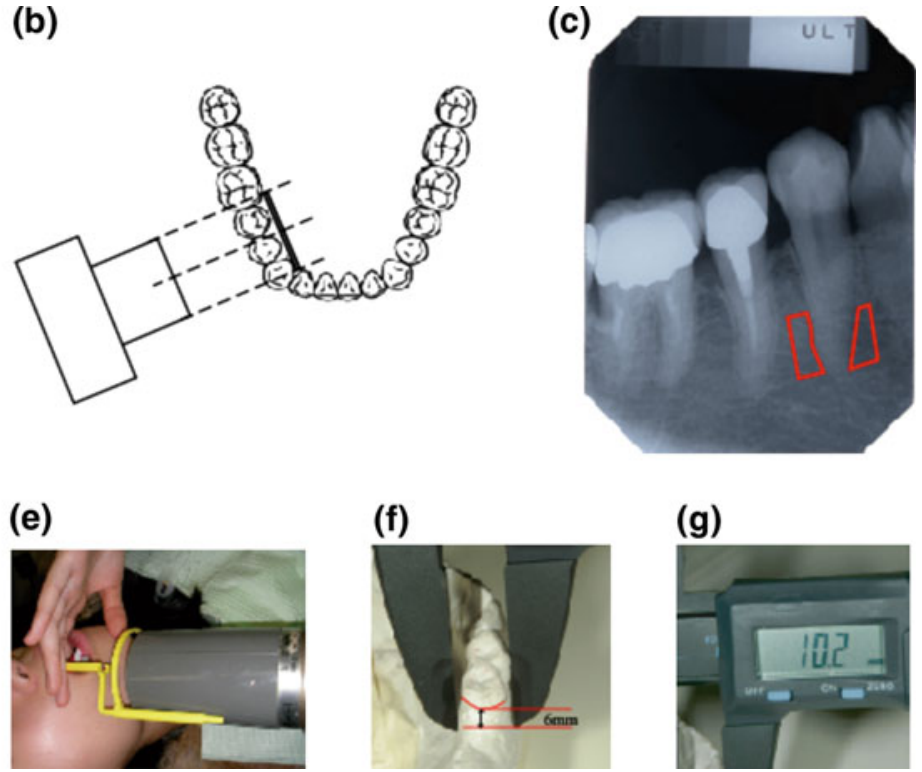

(f)

(g)

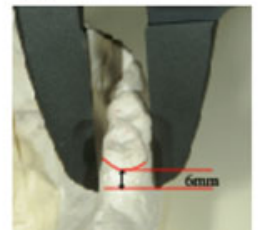

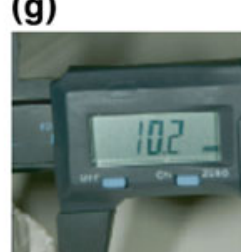

Fig. 1 Devices used for alveolar bone mineral density measurements. Dental X-rays were taken of the alveolar bone mineral density at the root of the first mandibular premolar tooth using purpose-designed image-editing software (No. PCT/jp2004/010815). a Pasting an aluminum step wedge to the film. b Illustration representing the

boundary of the cement-enamel junction and another halfway between the cement-enamel junction and the apex of the root. Lines were then dropped perpendicular to those at the mesial and distal spaces of the first premolar. The X-ray film density in the area of the resulting rectangle (or the region of interest [ROI]) was measured by first dividing the area into pixels, with sides of $1 / 1,534 \mathrm{~cm}$ in length. The brightness in each was then compared with a scale consisting of 256 grades of brightness (Fig. 1). To standardize the brightness and contrast among pictures taken on various occasions, an X-ray picture of the same tooth from a healthy control person was used as a reference. The histogram of the color bar on the reference picture was used for normalization, followed by calculation of the reference mean and standard deviation (Fig. 2). This method made it possible to accurately compare the placement of the X-ray apparatus against the right first premolar. c The lower half of the alveolar bone is circumscribed in red. $\mathbf{d}-\mathbf{g}$ Measurement taking of the buccolingual distance (alveolar bone mineral thickness) using a digital Nogis scale

brightness between individuals and images taken at different times. The use of an aluminum step wedge, not for a direct comparison of brightness between films, but for normalization and standardization of the data by computation, made it possible to achieve a coefficient of variation (CV) of $1.94 \%$ on a measurement of al-BMD in 30 subjects at 2-week intervals. Alveolar bone thickness (al-T), or the buccolingual distance, was measured manually with a digital Nogis scale.

\section{Chemical Assays}

Bacteriological studies on periodontal disease, including BANA test, were also carried out. Negative results were obtained, making the presence of active periodontal disease unlikely. Bone turnover markers, urinary 


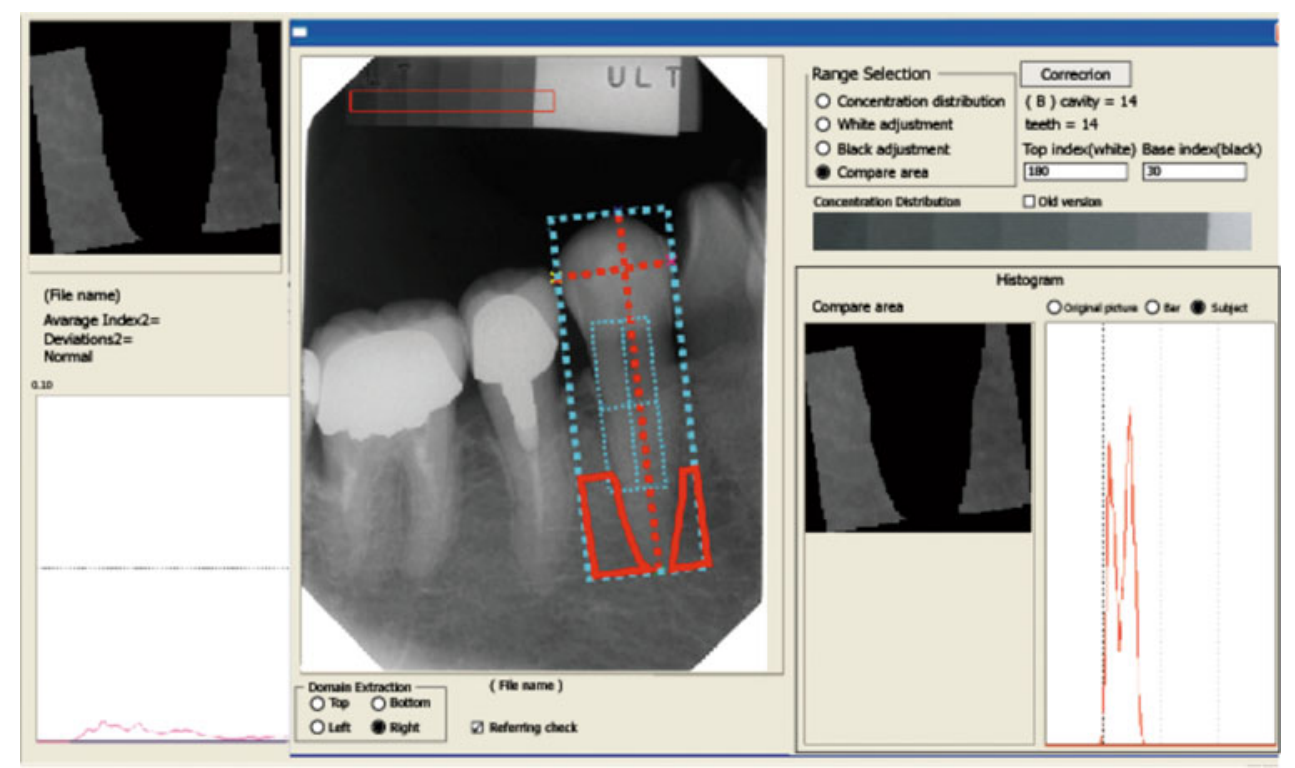

Fig. 2 Measurement and calculation of alveolar bone mineral density (al-BMD). To standardize the brightness and contrast among pictures taken on different days, an $\mathrm{X}$-ray was taken in a healthy person, and a histogram of the color bar on the reference picture was normalized, followed by calculation of the reference mean and standard variation. Lines were drawn from the apex of the root, parallel to the boundary of the cement-enamel junction, and halfway between the cement-enamel junction and the apex of the

deoxypyridinoline (DPD), and alkaline phosphatase were also measured. DPD was measured by high-speed liquid chromatography (HPLC), as previously described [32], and serum bone-specific alkaline phosphatase (BAP) was determined by immunoradiometric assay (IRMA), also as previously described [33].

\section{Statistical Analysis}

We arranged factors related to age, body size, bone size, and BMD in descending order, with the expectation that these factors would increase the fracture risk (Tables 1, 2). These potential risk factors were then compared with the actual incidence of fracture among the 30 female subjects. To examine the effect of age as a risk factor, age was presented in two measures: (1) '100-Age' (100 minus age) was used instead root. Lines were then also drawn perpendicular to those at the mesial and distal spaces of the first premolar. The X-ray film density in the area of the resulting rectangle was measured by first dividing the area into pixels with sides of $1 / 1,534 \mathrm{~cm}$ in length. The brightness in each pixel was compared with a scale consisting of 256 grades of brightness. Comparisons could then be made between films taken at different times for different individuals

of the actual patient age, and (2) '50-YSM' (50 minus number of years since menopause) was used instead of years since the onset of menopause; these parameters were used to make it easier to assess the influence of advancing age versus reductions in body size and bone density on osteoporosis. A correlation matrix was then constructed for these factors as well as the presence or absence of fracture (Table 3). A receiver operating characteristics (ROC) curve was then constructed to evaluate the contribution of each of these factors on the presence of fracture (Fig. 3), with calculation of area under the curve (AUC) and probability of asymptomatic significance (PAS). All statistical analyses were performed using SPSS ${ }^{\circledR} 10.13 \mathrm{~J}$ (SPSS Inc., Chicago, IL, USA) and Excel ${ }^{\circledR}$ Quantification version 3.0 (Esumi Co., Tokyo, Japan). The Mann-Whitney test was applied to 


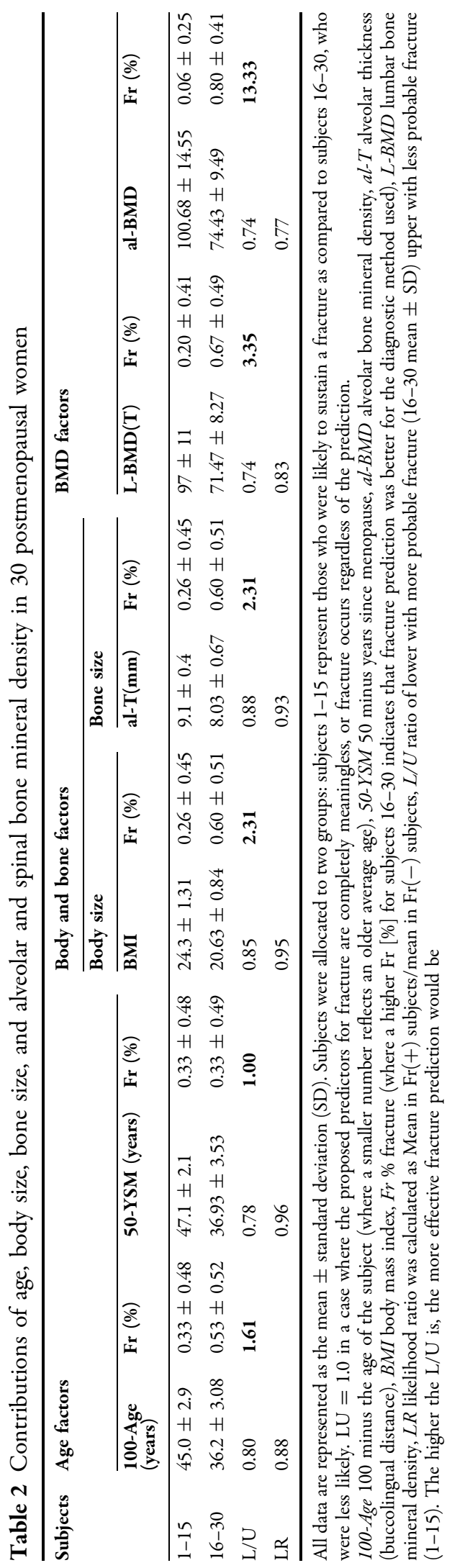

the data of L-BMD(T) and al-BMD, between the groups with and without fracture. Furthermore, contribution of L-BMD(T) or al-BMD on the occurrence of fracture was evaluated by multivariate discriminant analysis $\left(\right.$ Excel $^{\circledR}$ Quantification version 3.0).

\section{Prediction Formula for Fracture}

The prediction formula for fracture is as follows: $Y=a_{11} \quad x_{11}+a_{12} \quad x_{12}+a_{21} \quad x_{21}+a_{22} \quad x_{22}$, where $Y$ is the degree of fracture $(-1.5 \leq$ $Y \leq 1.5$ ).

$$
\begin{aligned}
& x_{11}, x_{12}, x_{21}, \text { and } x_{22} \text { are: } \\
& x_{11}: x_{11}=1(\mathrm{~L}-\mathrm{BMD}(\mathrm{T})<80), x_{11}=0(\mathrm{~L}-\mathrm{BMD}
\end{aligned}
$$

$$
x_{12}: x_{12}=1(\mathrm{~L}-\mathrm{BMD}(\mathrm{T}) \geq 80), x_{12}=0(\mathrm{~L}-\mathrm{BMD}
$$

(T) $<80)$

$$
x_{21}: x_{21}=1(\mathrm{al}-\mathrm{BMD}<84.9), x_{21}=0 \quad(\mathrm{al}-\mathrm{BMD}
$$
$\geq 84.9$ )

$$
x_{22}: x_{21}=1(\text { al-BMD } \geq 84.9), x_{12}=0(\text { al-BMD }
$$
$<84.9)$

$a_{11}, a_{12}, a_{21}$, and $a_{22}$ are the category weight scores indicating the degree of contribution of fracture.

\section{RESULTS}

All subjects, including those with fracture episodes, were arranged in the order of expected importance of risk factors: age factors (expressed as 100-Age and 50-YSM), body size (BMI) and bone size (al-T) factors, and BMD data (L-BMD[T] and al-BMD) (Tables 1, 2). This ranking took into account the expected increase in fracture incidence with advancing age, decreasing body and bone size, and decreasing BMD and alveolar trabecular thickness in the buccolingual region. Fracture occurrence (1) or not (0) was indicated for each risk factor. The 30 subjects were split into two 
Table 3 Correlation matrix constructed for various parameters

\begin{tabular}{|c|c|c|c|c|c|c|c|c|c|}
\hline \multirow{2}{*}{ Factors } & \multirow[t]{2}{*}{ 100-Age } & \multirow[t]{2}{*}{ 50-YSM } & \multicolumn{2}{|c|}{ Body and bone size factors } & \multicolumn{2}{|c|}{ BMD factors } & \multirow[t]{2}{*}{ BAP } & \multirow[t]{2}{*}{ DPD } & \multirow[t]{2}{*}{ Fracture } \\
\hline & & & BMI & al-T & L-BMD & al-BMD & & & \\
\hline 100-Age & 1 & $0.622^{* *}$ & 0.338 & $0.454^{*}$ & $0.604^{* *}$ & $0.456^{*}$ & -0.19 & -0.252 & $-0.447^{*}$ \\
\hline 50-YSM & & 1 & 0.257 & 0.146 & $0.476^{* *}$ & $0.373^{*}$ & -0.101 & -0.57 & -0.178 \\
\hline BMI & & & 1 & $0.413^{*}$ & 0.303 & 0.279 & -0.043 & -0.001 & -0.245 \\
\hline al-T & & & & 1 & $0.404^{*}$ & $0.401^{*}$ & -0.168 & -0.205 & $-0.403^{*}$ \\
\hline $\mathrm{L}-\mathrm{BMD}(\mathrm{T})$ & & & & & 1 & $0.702^{* *}$ & $-0.439^{*}$ & -0.237 & $-0.472^{* *}$ \\
\hline al-BMD & & & & & & 1 & $-0.439^{*}$ & -0.225 & $-0.614^{* *}$ \\
\hline BAP & & & & & & & 1 & 0.061 & 0.2 \\
\hline DPD & & & & & & & & 1 & $0.396^{*}$ \\
\hline Fracture & & & & & & & & & 1 \\
\hline
\end{tabular}

50-YSM 50 minus years since the onset of menopause, 100-Age 100 minus the age of the subject (where a smaller number reflects an older average age), al-BMD alveolar bone mineral density, al-T alveolar thickness (buccolingual distance), BAP bone alkaline phosphatase (enzyme immunoassay [EIA] normal range 7.9-29.9 U/L), BMI body mass index, DPD deoxypyridinoline (EIA normal range 2.8-7.6 in females), $L-B M D$ lumbar bone mineral density, $L-B M D(T)$ L-BMD T-scores

${ }^{*} P<0.05$

${ }^{* *} P<0.01$

groups for comparison: subjects 1-15 and subjects $16-30$. In the results, a higher fracture incidence in the latter group would indicate a higher predictability than the former group. A complete absence of predictability would give a value of 1.0. Table 1 also outlines the raw values for measurements of vitamin D $(25[\mathrm{OH}] \mathrm{D})$, follicle stimulating hormone (FSH), estradiol (E2), and parathyroid hormone (PTH) for each subject.

In the ROC analysis (Fig. 3), the PAS yielded significant values of 0.00006 for al-BMD and 0.007 for $\mathrm{L}-\mathrm{BMD}(\mathrm{T})$, respectively. From the results of the discriminant analysis, the category weight score was -0.275 in the group with an $\mathrm{L}-\mathrm{BMD}(\mathrm{T})<80$, and +0.189 in the group with an $\mathrm{L}-\mathrm{BMD}(\mathrm{T})$ of $\geq 80$. For al-BMD, the corresponding values were -0.860 with an alBMD <84.9, and +0.860 with an al-BMD of
284.9. In the discriminant analysis, we obtained the width of category scores, the distance between +0.860 and -0.860 or 1.720 for al-BMD, and the corresponding distance between +0.189 and -0.275 for $\mathrm{L}-\mathrm{BMD}(\mathrm{T})$. These results indicate a 1.720/0.464 $\approx 3.7$ times higher predictability of spinal fracture by al-BMD than L-BMD(T). By multivariate analysis, the accuracy of the rate of fracture, based on the two items of al-BMD and L-BMD(T), was $86.7 \%$ for al-BMD, which was higher than the corresponding value of $76.7 \%$ for L-BMD(T) (Fig. 4).

\section{DISCUSSION}

Numerous studies have been conducted to assess jaw BMD compared with other bony sites and to determine whether a relationship 


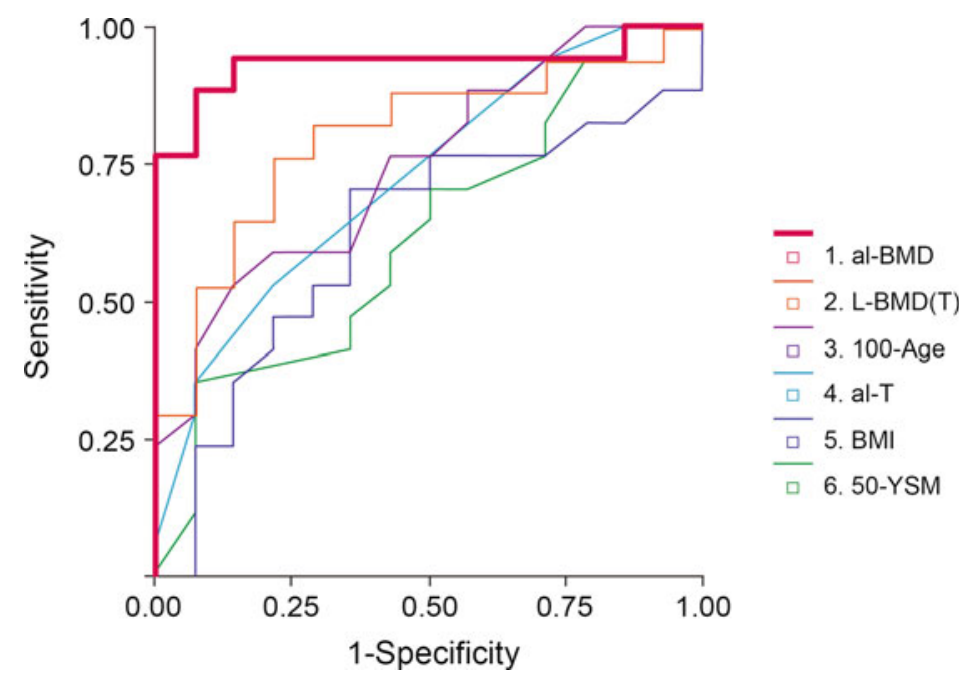

\begin{tabular}{|l|l|l|l|l|}
\hline $\begin{array}{l}\text { Fracture } \\
\text { predictors }\end{array}$ & $\begin{array}{l}\text { ROC curve } \\
\text { (AUC) }\end{array}$ & $95 \%$ CI & SE & $P$ value (PAS) \\
\hline al-BMD & 0.932 & $0.831-1.033$ & 0.052 & 0.00006 \\
\hline L-BMD(T) & 0.792 & $0.626-0.957$ & 0.084 & 0.007 \\
\hline $100-$ Age & 0.747 & $0.571-0.922$ & 0.090 & 0.0226 \\
\hline al-T & 0.710 & $0.710-0.992$ & 0.095 & 0.0516 \\
\hline BMI & 0.631 & $0.626-0.957$ & 0.107 & 0.2249 \\
\hline $50-$ YSM & 0.597 & $0.795-1.015$ & 0.106 & 0.3682 \\
\hline
\end{tabular}

Fig. 3 ROC curves assessing the contribution of alveolar and lumbar bone mineral density. PAS was high for both alveolar bone mineral density (al-BMD; 0.00006) and $\mathrm{L}-\mathrm{BMD}(\mathrm{T})(0.007)$. The association between al-BMD and fracture is evident. ROC curves for age (expressed as 100-Age in years), age after menopause (expressed as 50-YSM), alveolar bone thickness (al-T) as buccolingual distance measured by digital Nogis scale, and body mass

exists between osteoporosis and periodontal disease, tooth loss, and/or other disease states $[2-9,15,17-27]$. The aim of this study was to test the efficacy of measuring al-BMD using the Bone Right ${ }^{\circledR}$ method to predict fracture incidence. Using a multivariate analysis, as well as a correlation matrix and ROC analysis, we sought to determine an association index $(\mathrm{BMI})$ calculated as weight $(\mathrm{kg}) /$ height $(\mathrm{m})^{2} \times 100$. Contributions of 100-Age (0.023), al-T (0.052), 50-YSM (0.368), and BMI (0.225) were also significant. 100-Age 100 minus the age of the subject, 50-YSM age (years) since menopause, $A U C$ area under the curve, $P A S$ probability of asymptomatic significance, $R O C$ receiver operating characteristics on calculation of PAS, SE standard error of the mean

between the incidence of fracture and other potential risk factors, including age, body size, and bone size, using al-BMD and L-BMD measurements. We also aimed to determine whether measurements of alveolar bone density could predict bone loss and thereby the risk of osteoporotic fracture with more accuracy than measurements taken with 


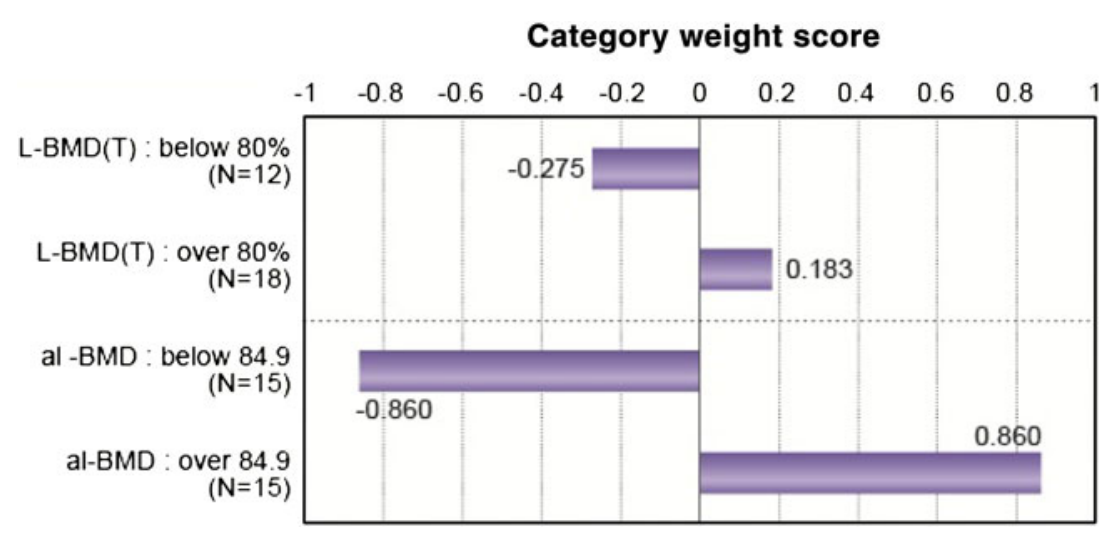

Fig. 4 Category score graph (accuracy rate 86.7\%). Category weight score represents the risk of $\mathrm{L}-\mathrm{BMD}(\mathrm{T})$ and al$\mathrm{BMD}$ to the fracture (presence or absence) as calculated by multivariate discriminant analysis. A multivariate analysis was used to compare the predictability of $\mathrm{L}-\mathrm{BMD}(\mathrm{T})$ and al-BMD to fracture using a category weight score method. Category weight score was -0.275 with an $\mathrm{L}-\mathrm{BMD}(\mathrm{T})$ of $<80 \%$, and +0.183 with $\geq 80 \%$. Category weight score was -0.860 with an al-BMD $<84.9$ (brightness), and it was +0.860 with al-BMD of $\geq 84.9$. Range is a guideline to

DXA of the lumbar vertebrae, L1-L4 (L-BMD[T]).

Our results showed that both al-BMD and $\mathrm{L}-\mathrm{BMD}(\mathrm{T})$ were closely related to the incidence of systemic fracture. Using the multivariate analysis, al-BMD had a fracture prediction accuracy rate of $86.7 \%$ compared with $76.7 \%$ for $\mathrm{L}-\mathrm{BMD}(\mathrm{T})$. This was determined using the prediction formula, as follows: $Y=a_{11} \quad x_{11}$ $(\mathrm{L}-\mathrm{BMD}[\mathrm{T}]<80)+a_{12} x_{12}(\mathrm{~L}-\mathrm{BMD}(\mathrm{T}) \geq 80)+a_{12}$ $x_{21}\left(\right.$ al-BMD <84.9) $+a_{22} \quad x_{22}($ al-BMD $\geq 84.9)$. For example, in subject 21, with an L-BMD(T) of 74 , an al-BMD of 80.07 , and an actual history of fracture, the category weight score was calculated as $Y=-0.275 \times 1-0.860 \times 1=$ -1.135 ; this indicates the highest risk of fracture for this patient. However, in subject 15 , where there was no history of fracture, $Y=1.043$, indicating a low risk of fracture.

While we generated a substantial amount of information in this study, we fell short of being able to predict the occurrence of systemic compare $\mathrm{L}-\mathrm{BMD}(\mathrm{T})$ and al-BMD. The range of $\mathrm{L}-\mathrm{BMD}(\mathrm{T})$ is $0.136| \pm| 0.275=0.456$. The range of al$\mathrm{BMD}$ is $0.860 \pm 0.860=1.720$. For fracture evaluation, al$\mathrm{BMD}$ is 3.7 times as heavily weighted as $\mathrm{L}-\mathrm{BMD}(\mathrm{T})$. Correlation of $\mathrm{L}-\mathrm{BMD}(\mathrm{T})$ and al-BMD to the fracture is $P<0.005$ and $P<0.001$. The ranges of L-BMD(T) and alBMD indicating the degree of predictability are 0.458 and 1.720 , approximately 3.7 times higher for al-BMD than for $\mathrm{L}-\mathrm{BMD}(\mathrm{T})$

fracture based on the BMD of the alveolar bone in this cohort of 30 postmenopausal women. However, the computed radiogrammetry developed by Takaishi et al. [28-30] has resulted in a remarkable step forward over previous attempts to gain an understanding of the association between jaw BMD, tooth loss, and osteoporotic fractures. The BMD measured at the right first molar tooth within the alveolar bone showed a highly significant regression with age (brightness $=166.8-1.3 \times$ age, $r=0.65, P<0.0001)$. The atypically high al$\mathrm{BMD}$ in this site was restricted to the sites of bisphosphonate-associated necrosis and postradiation-associated necrosis; the sensitivity of this method thus revealed links to necrosis in relation to subtle changes in bone loss, which had so far escaped detection using other methods [28]. This may be explained by an earlier onset and more rapid progression of bone loss in the alveolar bone than in the spinal column. 
One of the disadvantages of $\mathrm{L}-\mathrm{BMD}(\mathrm{T})$ measurements is the paradoxical rise in the apparent BMD along with degenerative changes to the spine, including osteoarthritis and disc degeneration, which begin to appear after middle age or following excessive and/or many years of intense exercise. This incidence of degenerative changes with advancing age can artificially increase the apparent BMD in these patients and interfere with the ability to make an accurate estimation of the risk of fracture. No such problem is found for alveolar bone measurements. Therefore, the early identification of jawbone loss would provide an effective 'alarm system' for identifying similar bone loss elsewhere in the body that may not be as easy to detect.

There were several limitations to this study. First, this study had a one-point retrospective observational design without an adequate follow-up. A prospective trial should be conducted before the utility of this method can be validated as a potential screening tool. Second, we were able to recruit only a small number of test subjects in this study, with just over half having sustained a fracture. While we recruited these patients unselectively and in chronological sequence, the fracture incidence in these subjects was remarkably high. This high incidence of fracture was unexpected, even with a Japanese cohort. Japan is known to have a higher overall incidence of fracture owing to the lifestyle and diet of the population. In 279 cases of postmenopausal women, $30.5 \%$ were observed to have a prevalent fracture, while $10.4 \%$ were observed to have incident fracture [34]. However, since epidemiological studies have previously focused on the incidence of hip and spinal fracture, it is possible that the actual incidence of fracture from minor accidents and falls could be under- represented. It is also possible that this discrepancy in fracture incidence as compared to other larger studies could stem from the small group of assembled patients in this study. Future studies would need a much larger prospective population-based study to confirm the findings in this study and avoid any potential geographical bias. Furthermore, future studies should include a control group that did not have osteoporosis to show a correlation between BMD measurements at the two sites. Third, the mean age of the patients in this cohort was rather young, and we did not assess older patients who were more prone to developing osteoporotic fractures. In addition, this method may encounter difficulty in identifying the location of the original alveolar bone in older, edentulous patients; this is of some concern, since the use of this technique is aimed at helping identify patients with osteoporosis, who are generally those of an advanced age and who have also lost some of their teeth. Fourth, future studies should compare another typical osteoporotic detection site, such as the hip, to compare the BMD between this and the alveolar bone. The study by Pluskiewicz et al. [22] showed good correlations between BMD changes in the hip and the mandible, and this would help confirm the reliability of alveolar bone for the detection of osteoporosis using the Bone Right $^{\circledR}$ method. In a similar vein, it would be useful to compare the results of the DXA scan with another method of imaging to further confirm the validity of our findings.

\section{CONCLUSION}

In conclusion, when compared with other risk factors (age, body size, bone turnover markers, and L-BMD[T]), al-BMD measurements showed a higher association with vertebral and long 
bone fractures than L-BMD and could successfully identify those patients who had sustained a fracture in the multivariate analysis. Our findings indicate that an assessment of alveolar bone density with Bone Right ${ }^{\circledR}$ may be a useful adjunct method for assessing patients of an advanced age during routine dental examinations to monitor the clinical picture and the potential course of osteoporosis.

\section{ACKNOWLEDGMENTS}

We thank Dr. Tim Werry, Dr. Rebecca Jackson, and Mrs. Yayoi Takaishi for editorial support in the preparation of this manuscript. No funding or sponsorship was received for this study or publication of this article. Dr. Yoshitomo Takaishi is the guarantor for this article and takes responsibility for the integrity of the work as a whole.

Conflict of interest. Dr. Yoshitomo Takaishi, Dr. Seizaburo Arita, Dr. Mitsugi Honda, Dr. Takeshi Sugishita, Dr. Aiko Kamada, Dr. Takashi Ikeo, Dr. Takami Miki, and Dr. Takuo Fujita declare no conflict of interest.

Compliance with Ethics Guidelines. All procedures followed were in accordance with the ethical standards of the responsible committee on human experimentation (institutional and national) and with the Helsinki Declaration of 1975 , as revised in 2000. Informed consent was obtained from all patients for being included in the study. This study was approved by the Institutional Review Board of Katsuragi Hospital.

\section{REFERENCES}

1. Jeffcoat MK. Osteoporosis: a possible modifying factor in oral bone loss. Ann Periodontol. 1998;3: $312-21$.

2. Martinez-Maestre MA, Gonzalez-Cejudo C, Machuca G, Torrejon R, Castelo-Branco C. Periodontitis and osteoporosis: a systematic review. Climacteric. 2010;13:523-9.

3. Krall EA, Garcia RI, Dawson-Hughes B. Increased risk of tooth loss is related to bone loss at the whole body, hip, and spine. Calcif Tissue Int. 1996;59: 433-7.

4. Krall EA, Dawson-Hughes B, Papas A, Garcia RI. Tooth loss and skeletal bone density in healthy postmenopausal women. Osteoporos Int. 1994; 4:104-9.

5. Groen JJ, Duyvensz F, Halsted JA. Diffuse alveolar atrophy of the jaw (non-inflammatory form of paradental disease) and pre-senile osteoporosis. Gerontol Clin. 1960;2:68-86.

6. Taguchi A, Suei Y, Ohtsuka M, Otani K, Tanimoto K, Hollender LG. Relationship between bone mineral density and tooth loss in elderly Japanese women. Dentomaxillofac Radiol. 1999;28:291-300.

7. Nicopoulou-Karayianni K, Tzoutzoukos P, Mitsea A, et al. Tooth loss and osteoporosis: the OSTEODENT study. J Clin Periodontol. 2010;36:190-7.

8. Megson E, Kapellas K, Bartold PM. Relationship between periodontal disease and osteoporosis. Int J Evid Based Healthc. 2010;8:129-39.

9. Habashneh RA, Alchalabi H, Khaden YS, Hazza'a AM, Odat Z, Johnson GK. Association between periodontal disease and osteoporosis in postmenopausal women in Jordan. J Periodontol. 2010;81:1613-21.

10. Kribbs PJ, Smith DE, Chesnut CH 3rd. Oral findings in osteoporosis. Part I: measurement of mandibular bone density. J Prosthet Dent. 1983;50:576-9.

11. Manzke E, Chesnut CH III, Wergedal JE, Baylink DJ, Nelp WB. Relationship between local and total bone mass in osteoporosis. Metabolism. 1975;24:605-15.

12. Klemetti E, Vainio P. Effect of bone mineral density in skeleton and mandible on extraction of teeth and clinical alveolar height. J Prosthet Dent. 1993;70:21-5. 
13. Earnshaw SA, Keating N, Hosking DJ, et al. Tooth counts do not predict bone mineral density in early postmenopausal Caucasian women. EPIC study group. Int J Epidemiol. 1998;27:479-83.

14. Jonasson G, Alstad T, Vahedi F, Bosaeus I, Lissner L, Hakeberg M. Trabecular pattern in the mandible as bone fracture predictor. Oral Surg Oral Med Oral Pathol Oral Radiol Endod. 2009;108:e42-51.

15. Verheij JG, Geraets WG, van der Stelt PF, et al. Prediction of osteoporosis with dental radiographs and age. Dentomaxillofac Radiol. 2009;38:431-7.

16. Jonasson G, Kiliaridis S, Gunnarsson R. Cervical thickness of the mandibular alveolar process and skeletal bone mineral density. Acta Odontol Scand. 1999;57:155-61.

17. Cakur B, Dagistan S, Sahin A, Harorli A, Yilmaz A. Reliability of mandibular cortical index and mandibular bone mineral density in the detection of osteoporotic women. Dentomaxillofac Radiol. 2009;38:255-61.

18. Vlasiadis KZ, Skouteris CA, Velegrakis GA, et al. Mandibular radiomorphometric measurements as indicators of possible osteoporosis in postmenopausal women. Maturitas. 2007;58:226-35.

19. Horner K, Karayianni K, Mitsea A, et al. The mandibular cortex on radiographs as a tool for osteoporosis risk assessment: the OSTEODENT Project. J Clin Densitom. 2007;10:138-46.

20. Yasar F, Akgunlu F. The differences in panoramic mandibular indices and fractal dimension between patients with and without spinal osteoporosis. Dentomaxillofac Radiol. 2006;35:1-9.

21. Taguchi A, Ohtsuka M, Tsuda M, et al. Risk of vertebral osteoporosis in post-menopausal women with alterations of the mandible. Dentomaxillofac Radiol. 2007;36:143-8.

22. Pluskiewicz W, Tarnawska B, Drozdzowska B. Mandibular bone mineral density measured using dual-energy X-ray absorptiometry: relationship to hip bone mineral density and quantitative ultrasound at calcaneus and hand phalanges. Br J Radiol. 2000;73:288-92.

23. Binte Anwar R, Tanaka M, Kohno S, et al. Relationship between porotic changes in alveolar bone and spinal osteoporosis. J Dent Res. 2007;86:52-7.

24. Miliuniene E, Alekna V, Peciuliene V, Tamulaitiene M, Maneliene R. Relationship between mandibular cortical bone height and bone mineral density of lumbar spine. Stomatologija. 2008;10:72-5.

25. Persson GR, Berglund J, Persson RE, Renvert S. Prediction of hip and hand fractures in older persons with or without a diagnosis of periodontitis. Bone. 2011;48:552-6.

26. Modano DE, Irigoyen ME, Borges-Yanez A, FloresSanchez I, Rotter RC. Osteoporosis, the risk of vertebral fracture, and periodontal disease in an elderly group in Mexico City. Gerodontology. 2011;28:19-27.

27. Okabe S, Morimoto Y, Ansai T, et al. Assessment of the relationship between the mandibular cortex on panoramic radiographs and the risk of bone fracture and vascular disease in 80-year olds. Oral Surg Oral Med Oral Pathol Oral Radiol Endod. 2008;106:433-42.

28. Takaishi Y, Ikeo T, Nakajima M, Miki T, Fujita T. A pilot case-control study on the alveolar bone density measurement in risk assessment for bisphosphonate-related osteonecrosis of the jaw. Osteoporos Int. 2010;21:815-25.

29. Takaishi Y, Okamoto Y, Ikeo T, et al. Correlations between periodontitis and loss of mandibular bone in relation to systemic bone changes in postmenopausal Japanese women. Osteoporos Int. 2005; 16:1875-82.

30. Takaishi Y, Ikeo T, Miki T, Nishizawa Y, Morii H. Suppression of alveolar bone resorption by etidronate treatment for periodontal disease: 4- to 5 -year follow-up of four patients. J Int Med Res. 2003;31:575-84.

31. Genant HK, Wu CY, van Kuijk C, Nevitt MC. Vertebral fracture assessment using a semiquantitative technique. J Bone Miner Res. 1993;8:1137-48.

32. Ohishi T, Takahashi M, Kawana K, et al. Age-related changes of urinary pyridinoline and deoxypyridinoline in Japanese subjects. Clin Invest Med. 1993;16:319-25.

33. Garnero P, Delmas PD. Assessment of the serum levels of bone alkaline phosphatase with a new immunoradiometric assay in patients with metabolic bone disease. J Clin Endocrinol Metab. 1993;77:1046-53.

34. Fukunaga M, Nakamura T, Shiraki M, et al. Absolute height reduction and percent height ratio of the vertebral body in incident fracture in Japanese women. J Bone Miner Metab. 2004;22:104-10. 